Revisiones

\title{
Factores de riesgo laboral para tenosinovitis del miembro superior
}

\section{Tenosynovitis upper extremity occupational risk factors}

\section{Mayra Mevic Garrafa Núñez', María Cristina García Martín², Graciela Sánchez Lemus³ \\ 1. Unidad Docente de Medicina del Trabajo. Madrid. Hospital Universitario de Móstoles. Madrid. España. \\ 2. Unidad Docente de Medicina del Trabajo. Madrid. Hospital Fundación Jiménez Díaz. Madrid. España. \\ 3. Unidad Docente de Medicina del Trabajo. Madrid. Hospital Universitario de Getafe. Madrid. España.}

\section{Recibido: 08-12-15}

Aceptado: 20-12-15

\section{Correspondencia}

Mayra Garrafa Núñez

Residente de Medicina del Trabajo

Servicio de Prevención de Riesgos Laborales

Hospital Universitario de Móstoles

C/Concepción 1-1C

28901 Getafe

Madrid. España.

Correo electrónico: mayramevic.garrafa@salud.madrid.org

Resumen

Objetivos: Identificar los factores de riesgo desencadenantes de tenosinovitis en los trabajadores verificando qué casos pueden ser considerados de origen laboral y reconocer las formas de presentación más frecuentes en relación al ámbito laboral.

Metodología: Se realiza una revisión bibliográfica de la literatura científica publicada en MedlinePubMed, Cochrane Library, EMBASE, CISDOC-ILO/OSH UPDATE, Scopus, LILACS e IBECS en el período comprendido entre 2007 a 2014.

Resultados: Se obtiene un total de 14 artículos entre los cuales 10 eran transversales y 4 son casos y controles. Todos ellos muestran una elevada probabilidad de desarrollar tenosinovitis en el ámbito laboral. La relación con movimientos repetitivos fue el factor de riesgo más importante con OR $>2$, seguido de posturas forzadas y el uso de herramientas vibratorias. Respecto a los factores psicosociales la depresión presenta una $\mathrm{OR}=3,04$ (IC95\% 2,43-3,81) y la combinación de movimiento repetitivo y el estrés, OR = 4,94 (IC95\% 3,532-6,91).

Conclusión: Movimientos repetitivos, posturas forzadas y mantenidas, herramientas vibratorias, edad, ser mujer, raza blanca, estrés fueron los factores de riesgo encontrados sin poder demostrar en ninguno causalidad, siendo los trastornos musculoesqueléticos más prevalentes el síndrome del manguito de los rotadores, la enfermedad de De Quervain, dedo en gatillo y la epicondilitis lateral y medial.

$$
\text { Med Segur Trab (Internet) 2015; } 61 \text { (241) 486-503 }
$$

Palabras clave: Tenosinovitis, factores de riesgo, lesiones ocupacionales, relacionado al trabajo, enfermedades ocupacionales. 


\section{Abstract}

Objectives: To identify the workers tenosynovitis triggering risk factors verifying which can be considered work-related cases and recognizing the most frequently occurring form in the workplace.

Methods: A literature review of the scientific literature published in PubMed-Medline, Cochrane Library, EMBASE, CISDOC-ILO/OSH UPDATE, Scopus, LILACS and IBECS over the period 2007-2014 is performed.

Results: A total of 14 items were obtained, among which 10 were cross-sectional items and 4 cases and controls. They all show a high probability of developing tenosynovitis at the workplace. The relationship with repetitive movements was the most important risk factor with OR $>2$ followed by awkward postures and vibrating tools use. Regarding psychosocial factors of depression in an OR $=3.04$ (CI95\% 2.43-3.81) and the combination of repetitive motion and stress, $\mathrm{OR}=4.94$ (CI95\% 3.532 to 6.91).

Conclusion: Repetitive movements, enforced and maintained postures, vibrating tools, age, being female, white race, stress were the risk factors found, however it was not possible to demonstrate if it was merely coincidence. The most prevalent musculoskeletal disorders were rotator cuff syndrome, Quervain's disease, trigger finger and the lateral and medial epicondylitis.

Med Segur Trab (Internet) 2015; 61 (241) 486-503

Keywords: Tenosynovitis, risk factors, occupational injuries, work-related, occupational diseases. 


\section{INTRODUCCIÓN}

Los trastornos musculoesqueléticos de origen laboral son alteraciones que sufren estructuras corporales como los músculos, articulaciones, tendones, ligamentos, nervios, huesos y el sistema circulatorio, causadas o agravadas fundamentalmente por el trabajo y los efectos del entorno en el que este se desarrolla ${ }^{1}$. Se encuentran entre los problemas más importantes de salud en el trabajo y causan impacto sobre la calidad de vida con un coste anual elevado. La forma de manifestación más frecuente son molestias o dolor local y restricción de la movilidad, que pueden obstaculizar el rendimiento normal en el trabajo o en otras tareas de la vida diaria ${ }^{1}$.

Las tenosinovitis de origen laboral pueden ser originadas por el tipo de trabajo que se desarrolla, bien como consecuencia de un accidente laboral o como una lesión que se aparece sobre una patología de base previamente existente que se ve agravada por la actividad física desempeñada durante la jornada de trabajo, en la mayoría de los casos es difícil distinguir si se trata de una patología nueva o si se trata de una patología que aparece a consecuencia de las actividades laborales sobre una lesión de base ya existente, además en la mayoría de los casos es difícil identificar un único factor causal $^{3}$.

En la Unión Europea las enfermedades musculoesqueléticas son un problema de salud creciente y significativa. Más de 40 millones de trabajadores en Europa se ven afectados lo que representa un costo estimado para la sociedad de entre el 0,5 y el 2 por ciento del producto interior bruto (PIB) ${ }^{4}$.

En cuanto a la normativa legal en España, el artículo 4 de la Ley de Prevención de Riesgos Laborales define como «riesgo laboral» la posibilidad de que un trabajador sufra un determinado daño derivado del trabajo. A su vez se considerarán como «daños derivados del trabajo" las enfermedades, patologías o lesiones sufridas con motivo u ocasión del trabajo ${ }^{5}$. Estos trastornos musculoesqueléticos están codificados como enfermedades profesionales del grupo 2 correspondiente a agentes físicos del Anexo 1 del Cuadro de enfermedades profesionales, (códigos 01 2D0101, 01 2D0201 y 01 2D0301).

La Organización Internacional del Trabajo (OIT) define que la enfermedad profesional contiene dos elementos principales: la relación causal entre la exposición en un entorno de trabajo o actividad laboral específicos, y una enfermedad específica, y el hecho de que, dentro de un grupo de personas expuestas, la enfermedad se produce con una frecuencia superior a la tasa media de morbilidad del resto de la población ${ }^{7}$.

En España la incidencia de estas patologías es muy elevada con respecto al total de las enfermedades profesionales reportadas. Un estudio descriptivo realizado por el Instituto Nacional de Seguridad e Higiene en el Trabajo (INSHT) entre los periodos 2007-2012 muestra que en los partes cerrados de enfermedades profesionales notificadas al Centro de Estudios y Promoción Social (CEPROSS), los diagnósticos más frecuentes fueron la epicondilitis lateral con un $22,4 \%$ sobre el total, el síndrome del túnel carpiano con un $15,1 \%$ y las tenosinovitis de mano y muñeca con el 13,4\%. Además en relación con los días de baja totales, establece que el diagnóstico que aportó mayor número de días acumulados por incapacidad laboral fue la epicondilitis lateral con un total de más de 1,2 millones de días totales, seguido del síndrome del túnel carpiano con más 860 mil y las tendinitis de mano-muñeca con unos $600 \mathrm{mil}^{8}$.

Según los últimos datos publicados en el CEPROSS-2 en 2015, sobre el número de partes comunicados en el periodo comprendido entre enero a diciembre del 2014 , de un total de 17378 partes reportados sobre enfermedades profesionales, 8476 partes (48,7\%) corresponden a las enfermedades del grupo 2D (códigos 01 2D0101, 01 2D0201 y $012 \mathrm{D} 0301$ ) y de estos $23,9 \%$ causaron baja y los restantes $24,9 \%$ no causaron baja (56,11\% hombres y 43,88\% mujeres), lo que confirma la importancia de este estudio'.

Dado que en muchas ocasiones es difícil establecer la causa de las tenosinovitis de origen laboral, a partir de una revisión bibliográfica de la literatura científica más reciente, 
el presente trabajo tienen por objeto identificar los posibles factores de riesgo desencadenantes de tenosinovitis en trabajadores y en qué casos puede asociarse a causas de origen laboral, así como describir el impacto de la enfermedad por su frecuencia de presentación el ámbito laboral.

\section{MATERIAL Y MÉTODOS}

Se realiza una revisión bibliográfica de la literatura científica publicada entre 2007 y 2014 llevada a cabo en noviembre de éste último año en las principales bases de datos bibliográficas: Medline-PubMed, Cochrane Library, EMBASE, CISDOC-ILO/OSH UPDATE, Scopus, LILACS e IBECS mediante los siguientes descriptores MeSH y DeCS: tenosynovitis, occupations, occupational injuries, cumulative trauma disorders, causality, risk factors, work-related, ocupational diseases y utilizando los siguientes filtros: texto completo, humanos, idiomas: español, italiano e inglés, y años: 2007-2014. La estrategia de búsqueda se completó con búsqueda libre utilizando como términos de la misma: tenosynovitis, risk factors, cumulative trauma disorders, occupational diseases.

Se definieron los siguientes criterios de inclusión y exclusión:

\section{Criterios de inclusión}

- Estudios en humanos de ambos sexos, en población trabajadora sin establecer límite de edad, escritos en inglés, italiano y español, publicados entre 2007-2014, con resumen y texto completo disponible y estudios de tenosinovitis/tendinitis de miembro superior incluyendo enfermedad de De Quervain, dedo en gatillo, síndrome manguito de los rotadores y epicondilitis.

\section{Criterios de exclusión}

- Estudios de diagnóstico, tratamiento conservador, tratamiento quirúrgico, técnicas diagnósticas, rehabilitación, pacientes con enfermedades de base (DM, artritis reumatoide, síndrome del túnel del carpo (STC) o tenosinovitis infecciosa), artículos de opinión, cartas al editor, libros, protocolos, comunicaciones, a propósito de un caso, notas técnicas, revisión de expertos, guías de práctica clínica.

Sobre la colección bibliográfica se realizó un análisis de pertinencia con el fin de verificar la idoneidad de los artículos para ser incluidos el estudio, y se tuvieron en cuenta las guías SIGN como ayuda para determinar el nivel de evidencia. 


\section{RESULTADOS}

Inicialmente se obtuvo una colección de 161 artículos de la que fueron excluidos 147 artículos por no cumplir los criterios de inclusión o presentar alguna característica de los criterios de exclusión, resultando finalmente 14 artículos seleccionados para ser incluidos en este trabajo de revisión (tabla I).

Tabla I. Resultados de la búsqueda bibliográfica y resultado del proceso de selección de artículos en base a criterios de inclusión/exclusión y pertinencia

\begin{tabular}{|c|c|c|c|c|c|}
\hline Base de datos & Ecuaciones de búsqueda & $\begin{array}{l}\text { Artículos } \\
\text { encontrados }\end{array}$ & $\begin{array}{c}\text { Artículos } \\
\text { descartados }\end{array}$ & $\begin{array}{l}\text { Artículos } \\
\text { redundantes }\end{array}$ & $\begin{array}{c}\text { Artículos } \\
\text { seleccionados }\end{array}$ \\
\hline PubMed & $\begin{array}{l}\text { ((«Tenosynovitis»[Mesh]) AND } \\
(\text { («Occupations»[Mesh] OR } \\
\text { («Occupational Injuries»[Mesh] } \\
\text { OR ("Cumulative Trauma } \\
\text { Disorders»[Mesh] OR } \\
\text { («Causality»[Mesh] OR («Risk } \\
\text { Factors»[Mesh] OR»work-related»[All } \\
\text { Fields]) AND ("Occupational } \\
\text { Diseases»[Mesh])))) AND } \\
\text { («2007»[Date - Publication]: } \\
\text { "2014»[Date - Publication])) NOT } \\
\text { "treatment»)) Filters: Full text, } \\
\text { Humans }\end{array}$ & 67 & 58 & 1 & 8 \\
\hline EMBASE & $\begin{array}{l}\text { ('tenosynovitis'/exp OR } \\
\text { 'tenosynovitis' AND } \\
\text { ('occupational diseases'/exp OR } \\
\text { 'occupational } \\
\text { diseases') AND ('causality' OR } \\
\text { 'occupations' OR } \\
\text { 'occupational injuries' OR } \\
\text { 'cumulative trauma disorders' OR } \\
\text { 'risk factors' OR 'work related stress') } \\
\text { AND [2007-2014]/py AND [embase]/ } \\
\text { lim)) OR 'tenosynovialitis') }\end{array}$ & 63 & 63 & - & 0 \\
\hline $\begin{array}{l}\text { CISDOC } \\
\text { ILO/OSH } \\
\text { UPDATE }\end{array}$ & Tenosynovitis & 22 & 21 & - & 1 \\
\hline $\begin{array}{l}\text { Otras } \\
\text { fuentes }\end{array}$ & & 9 & 4 & - & 5 \\
\hline TOTAL & & 161 & 146 & 1 & 14 \\
\hline
\end{tabular}

De un total de 14 artículos, 4 eran de casos y controles y 10 estudios transversales.

La tabla II presenta los principales hallazgos encontrados en los diferentes trabajos incluidos en el estudio, clasificados por el tipo de estudio y año de publicación. 


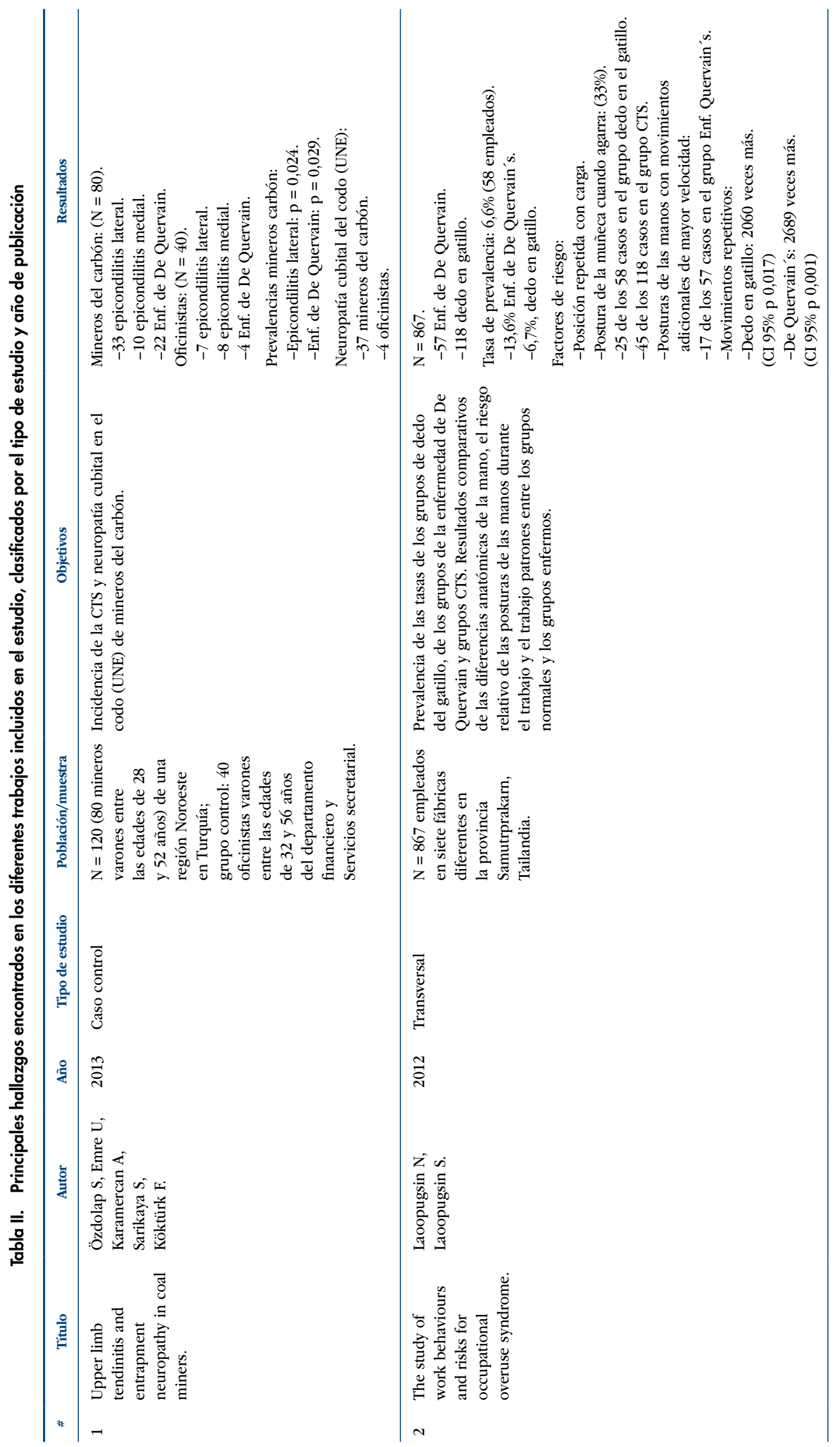




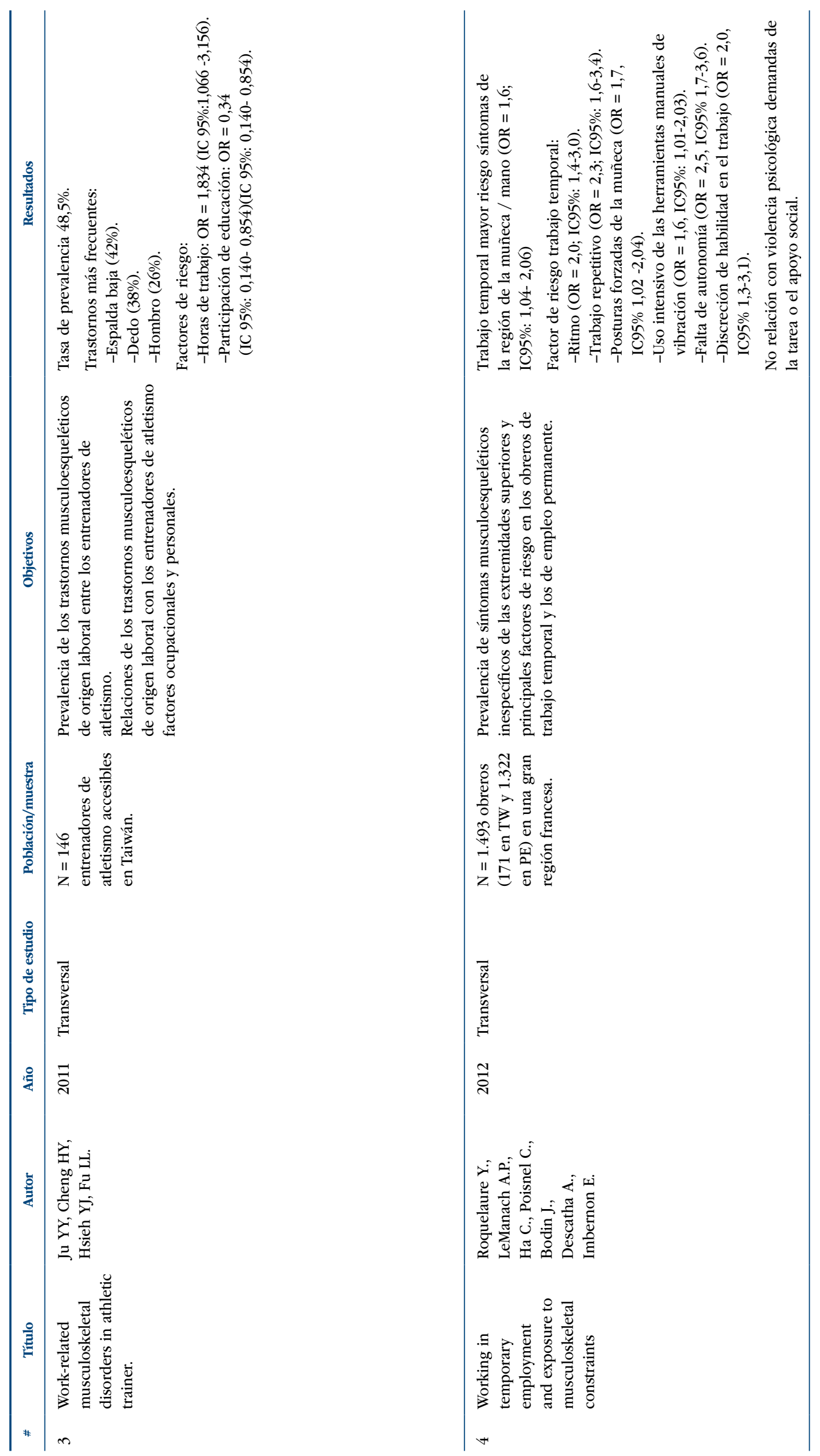




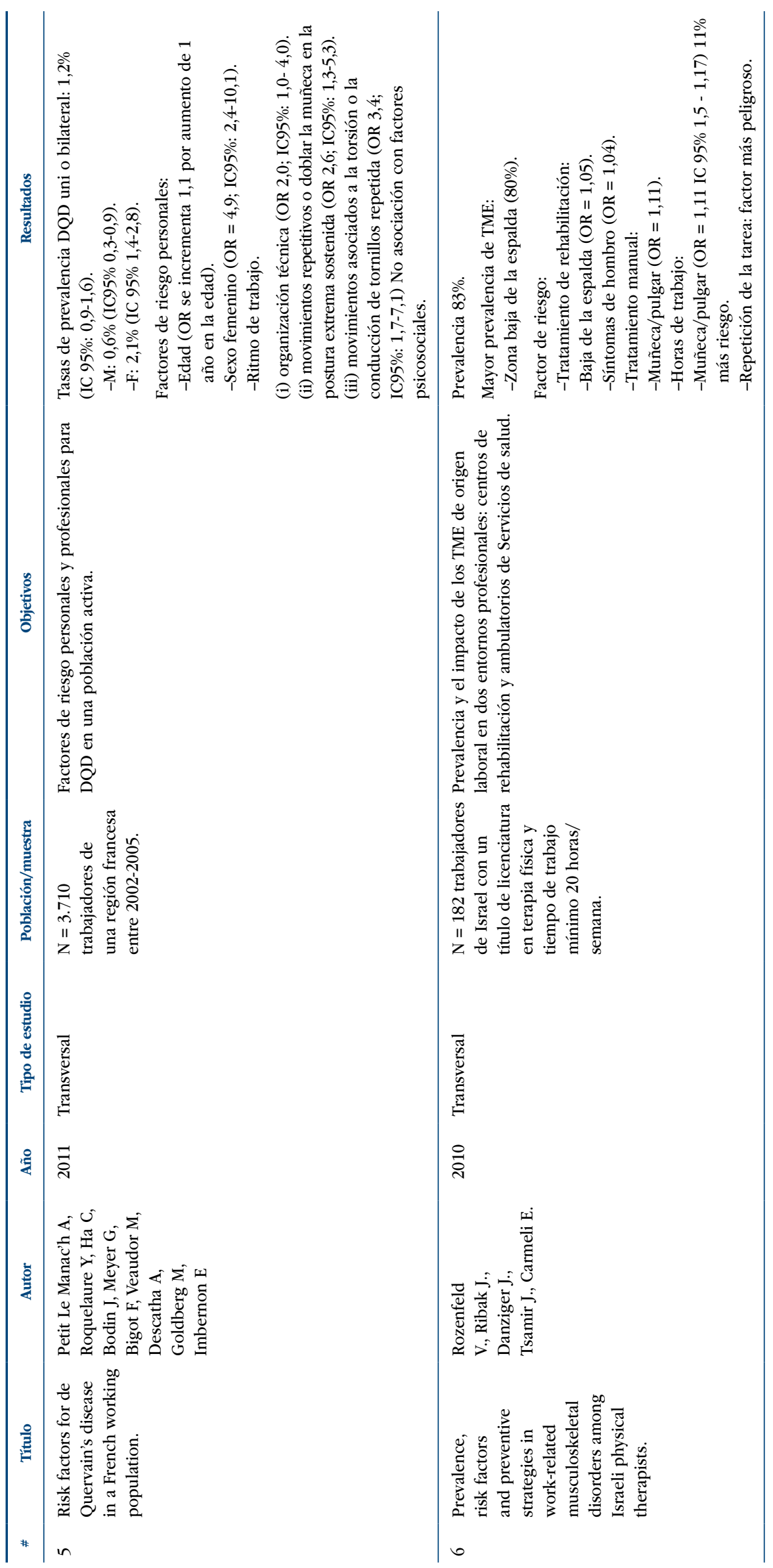




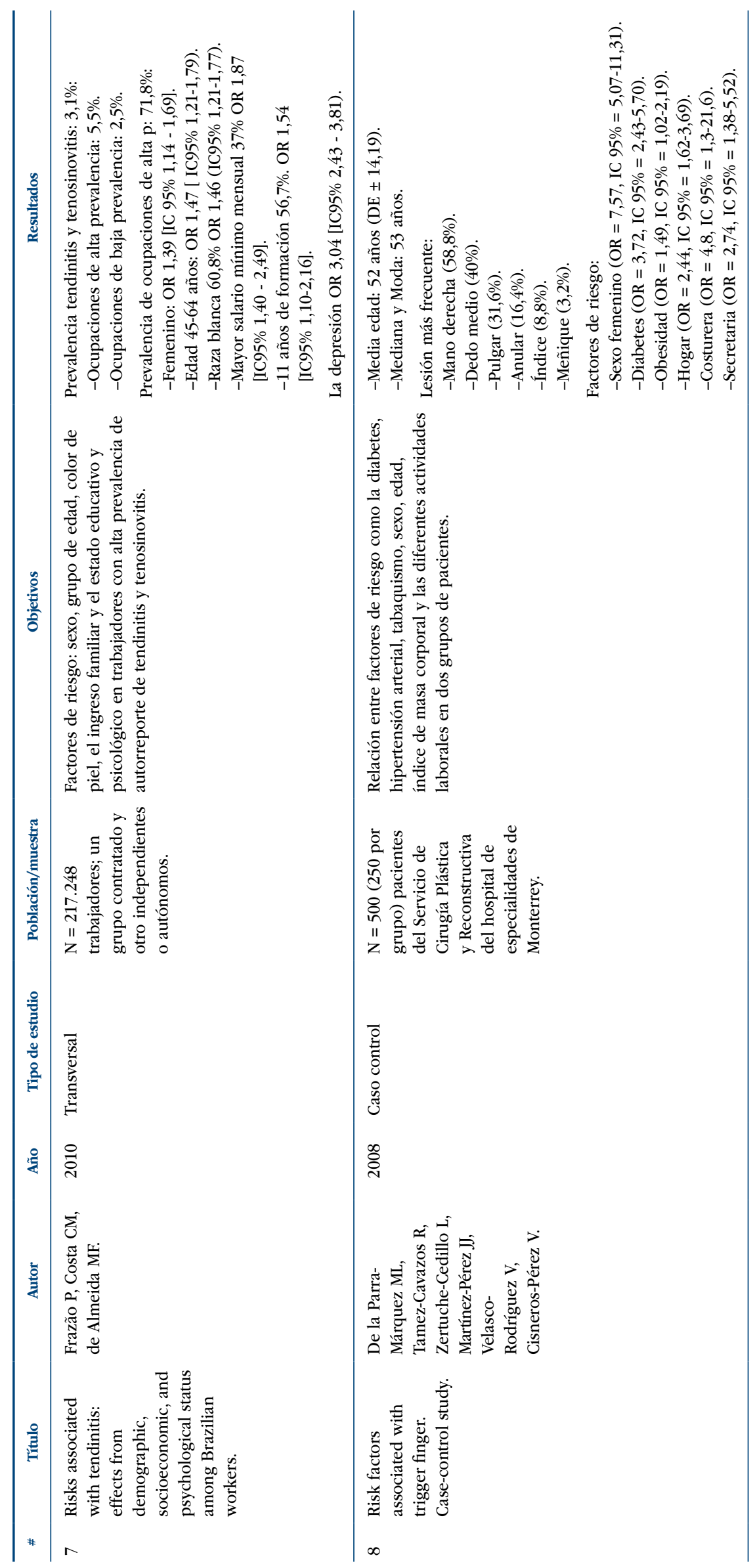




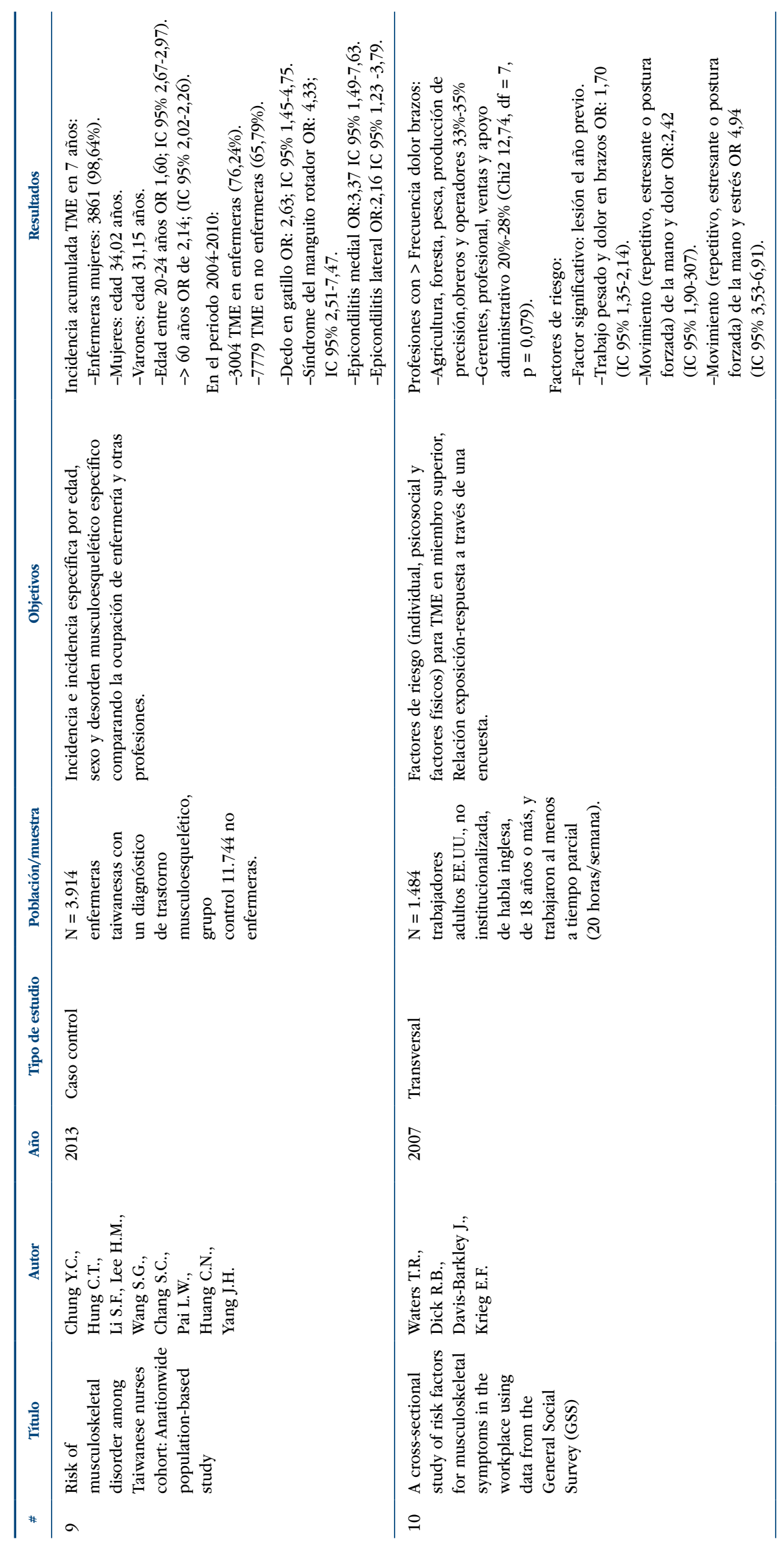




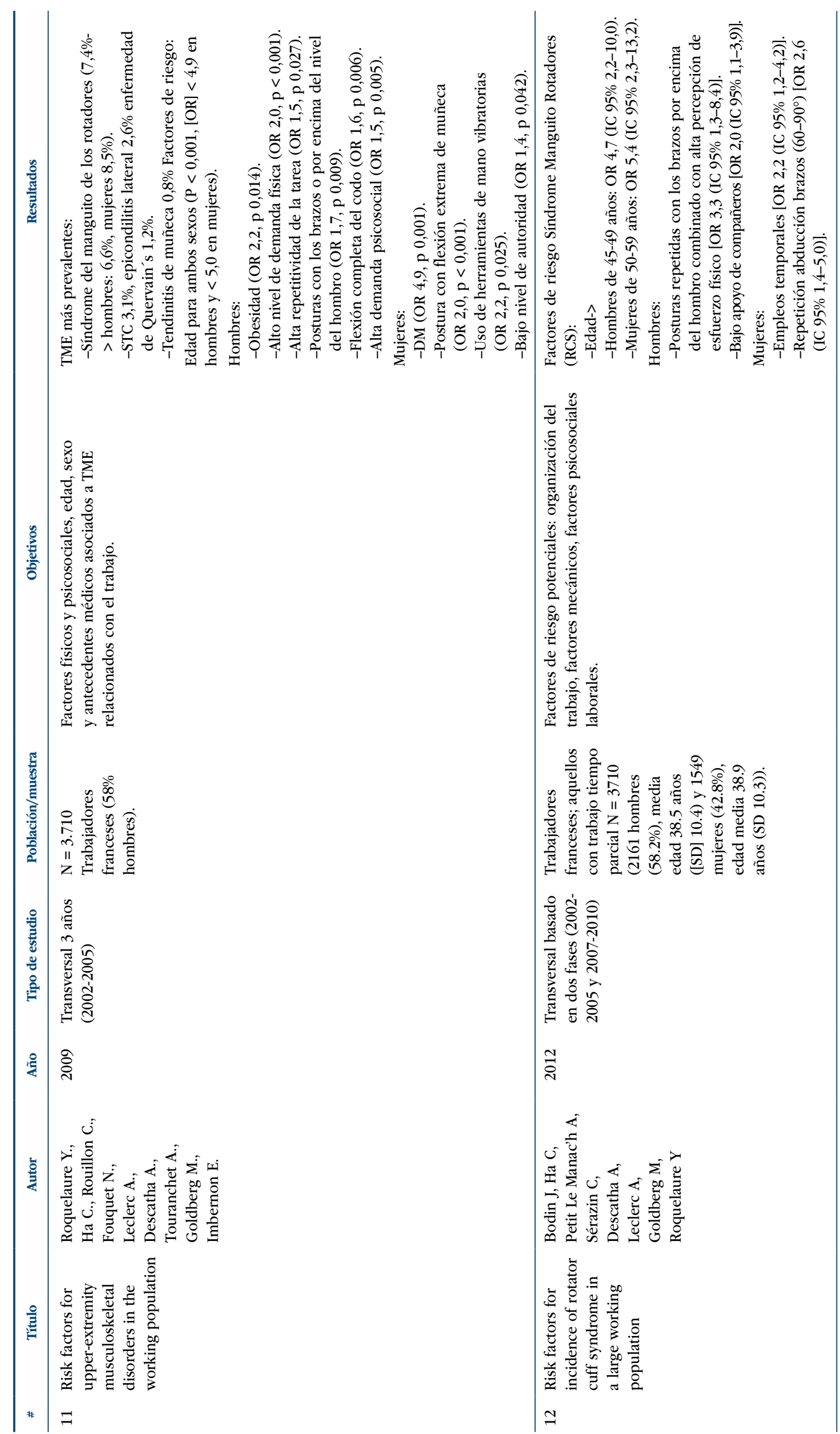




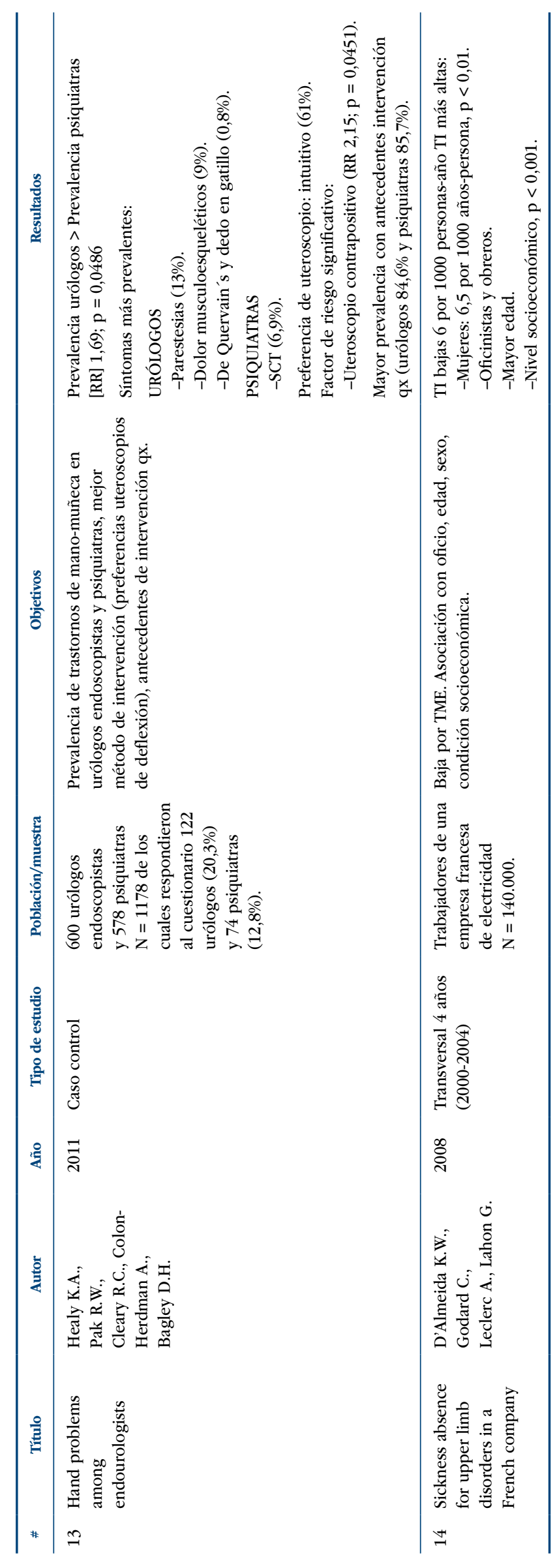


A partir de los resultados de los diferentes artículos que componen el estudio, se identificaron diferentes factores de riesgo de tipo laboral como son los movimientos repetitivos, posturas forzadas extremas, utilización intensiva de herramientas manuales vibratorias, ritmo y número de horas de trabajo, los asociados a factores psicosociales y a factores de riesgo individuales como edad, sexo, raza, nivel socioeconómico y su asociación a otras patologías de base y a otras tendinitis, para los que a continuación se presentan los principales resultados.

\section{Tenosinovitis en relación a factores de riesgo laboral}

Movimiento repetitivo: Laoopusing et al. (2012) en un estudio que tenía por objetivo proporcionar datos clínicos sobre la prevalencia y factores de riesgo para el desarrollo de dedo en gatillo y enfermedad de De Quervain observó que los movimientos repetitivos eran capaces de incrementar en 2.060 veces la posibilidad de presentar un dedo en gatillo y 2.689 en la enfermedad de De Quervain que cuando no se encuentra sometido a este tipo de movimiento. ${ }^{10}$ Roquelaure $C$. et al. (2012) para el riesgo de encontrase sometido a trabajo repetitivo, obtiene valores de OR $=2,3$ (IC95\% 1,6- 3,4) ${ }^{11}$. Petit Le Manac'b et al. en un estudio transversal que incluyó 3.710 trabajadores de una región francesa encontró una OR = 2,6 (IC95\% 1,3- 5,3) para los movimientos repetitivos o doblar la muñeca en la postura extrema sostenida. ${ }^{12}$ Roquelaure et al. (2009) en 3.710 trabajadores expuestos a una alta repetitividad de la tarea, obtuvo una OR $=1,5$, $\mathrm{p}=0,027 .{ }^{13}$ Bodin J. et al. (2012) una OR $=2,6$ para desarrollar el síndrome del manguito de los rotadores ${ }^{14}$. Rozenfeld $V$ et al. (2010) en un artículo en el que intentan determinar la prevalencia y el impacto de los trastornos musculoesqueléticos de origen laboral en 182 fisioterapeutas en Israel, concluyó que la repetición de la tarea es el factor más peligroso como desencadenante ${ }^{15}$. Waters T.R. et al. en un estudio transversal realizado en los EE.UU., estableció una asociación de movimiento repetitivo y dolor obteniendo una $\mathrm{OR}=2,42(\mathrm{IC} 95 \% 1,90-3,07)$ y para la combinación de movimiento repetitivo y estrés una OR $=, 94\left(\right.$ IC95\% 3,53-6,91).$^{16}$

Posturas forzadas extremas: Laoopugsin et al. (2012) realizó en Tailandia un estudio transversal en el que 25 de los 58 casos que presentaron dedo en gatillo estuvieron expuestos a posturas forzadas extremas y lo mismo para 45 de los 118 casos que presentaron síndrome del túnel del carpo $^{10}$.

Roquelaure $Y$. et al. (2012) realizaron un estudio transversal que incluyó 1.493 obreros, obteniendo una OR = 1,7 (IC95\% 1,02-2,0) para posturas forzadas de la muñeca en trabajadores con empleo temporal en relación a los que tenían empleo permanente ${ }^{11}$ al igual que en un estudio anterior realizado por los mismos autores, en el que obtuvieron una OR $=2$ con $\mathrm{p}<0,001$. $^{13}$ Petit Le Manac'b et al. (2011) encuentran una OR $=2,6$ (IC95\% 1,3-5,3) al mantener la muñeca en una postura extrema sostenida, ${ }^{12}$ finalmente Healy K.A (2011) en un estudio de casos y controles con 600 urólogos endoscopistas que realizaban procedimientos laparoscópicos prolongados encontró que aumentaron los riesgos para trastornos musculoesqueléticos con significación estadística ${ }^{17}$.

Uso intensivo de herramientas manuales vibratorias: El número de estudios que encuentren asociación entre este factor de riesgo y la tenosinovitis es menor, Laoopugsin et al. (2012) encontró 17 casos de 57 en el grupo de enfermedad de De Quervain que estuvieron expuestos a posturas de las manos con movimientos adicionales de mayor velocidad, ${ }^{10}$ Roquelaure $Y$ et al.(2012) obtuvieron una OR $=1,6$ (IC95\% 1,01-2,03) ${ }^{11}$ en cordancia con los resultados obtenidos en estudio previo que publicó en el 2009 donde describieron una OR $=2,2 ; \mathrm{p}=0,025 .{ }^{13}$

Ritmo y número de horas de trabajo: Ju YY et al. (2011) al estudiar la prevalencia de los trastornos musculoesqueléticos de origen laboral en los entrenadores de atletismo, encontró como factor de riesgo el número de horas de trabajo con una OR = 1,8 (IC95\% 1,06- 3,15), ${ }^{18}$ y Petit Le Manac'h et al. (2011) lo asoció al ritmo de trabajo y la organización técnica OR $=2,0\left(\right.$ IC95\% 1,0- 4,0). ${ }^{12}$ 


\section{Tenosinovitis en relación a factores psicosociales}

Entre los principales factores psicosociales que han sido estudiados en una posible relación con las tenosinovitis, podemos citar la autonomía/autoridad, apoyo de compañeros y demanda psicosocial.

Roquelaure Y et al. 2009 en un estudio cuyo objetivo era evaluar la asociación de trastornos musculoesqueléticos y alta demanda psicosocial laboral obtuvo una OR $=1,5$, $\mathrm{p}=0,005$ en concordancia con el bajo nivel de autoridad que obtuvo una OR $=1,4$, $\mathrm{p}=0,042 .{ }^{13} \mathrm{El}$ mismo autor en el 2012 realizó un estudio en 1493 obreros, intentando comparar la prevalencia de síntomas musculoesqueléticos inespecíficos de las extremidades superiores y sus principales factores de riesgo los obreros contratados a través de empresas de trabajo temporal con los que tenían empleo permanente, y al igual que Petit Le Manac'b (2011) ${ }^{12}$ no encontraron relación con la demanda psicológica, sobrecarga de tareas o la falta de apoyo social. ${ }^{11}$ Waters T.R. et al. en un estudio transversal con 1.484 trabajadores encontró una OR = 4,94 (IC95\% 3,53-6,91) de posturas forzadas de la mano en relación al movimiento repetitivo y combinarlo con la variable estrés. ${ }^{16}$

El tipo de contrato parece también contribuir a incrementar el riesgo, Frazão P et al. (2010) en un estudio transversal que incluyó 217.248 trabajadores brasileños agrupados en dos grupos, unos contratados formalmente que recibían honorarios mensuales y otros independientes o autónomos que cobraban por acto de servicio, encontró que los riesgos asociados a tendinitis fueron del 37\% mayores para los que recibían mayor salario mensual con una OR = 1,87 (IC95\% 1,40-2,49) al igual que los que tuvieron más de 11 años de formación con un 56,7\% (OR = 1,54; IC95\% 1,10-2,16), todo esto posiblemente relacionado con que los primeros estuvieron expuestos a largas horas de trabajo, descansos insuficientes, y las metas de producción establecidas por el empleador ${ }^{19}$.

\section{Tenosinovitis y su relación con factores de riesgo individuales}

Edad: Frazao et al. (2010), encontró un riesgo mayor para el grupo de edad de 45-64 años respecto al de 25-44 años (OR = 1,47 IC95\% 1,21-1,79). ${ }^{19}$ Chung $Y$ et al. (2013) de forma similar estableció dos grupos de trabajadores uno entre 20-24 y otro de mayores de 60 años encontrado una OR de 1,60 y 2,14, respectivamente y con diferencia estadísticamente significativa. ${ }^{20}$. Roquelaure et al. (2009 también encuentra una relación entre el riesgo y la edad con una $\mathrm{OR}=4,9$ para hombres y $\mathrm{OR}=5$ para mujeres con una $\mathrm{p}<0,00,1^{13}$ dichos estudios confirman la importancia de la edad como factor importante de riesgo personal. Petit Le Manac et al. (2011) en un estudio transversal con 3.710 trabajadores seleccionados aleatoriamente en una región francesa, encontró que los riesgos personales y ocupacionales para desarrollar enfermedad de De Quervain en población activa se incrementaban con una OR de 1,1 por cada año que aumentaba la edad. $^{12}$

Sexo: Roquelaure et al. (2009) en un estudio transversal realizado en Francia encontró una prevalencia del 8,5\% para mujeres y 6,6\% para hombres ${ }^{13}$ en el síndrome del manguito de los rotadores, datos similares a los que describe Bodin J. et al.(2012) ${ }^{14}$. Petit Le Manac et al. (2011) realizaron también un estudio transversal con 3.710 trabajadores de una región francesa, estudiando la prevalencia y encontrando que la enfermedad de De Quervain fue mayor para las mujeres con 2,1\% (IC 95\% 1,4-2,8) y para hombres con 0,6\% (IC95\% 0,3-0,9), presentando las mujeres un riesgo muy superior al de los hombres, con una OR de 4,9 (IC95\% 2,4-10,1). ${ }^{12}$ Frazao et al. (2010) obtuvo datos similares con una OR = 1,39 (IC95\% 1,14-1,69) $)^{19}$ y Chung Y et al. (2013) observan que la incidencia acumulada de trastornos musculoesqueléticos para las enfermeras mujeres en un periodo de 7 años frente al de los hombres fue del 98,64\%. ${ }^{20}$

Raza: Un estudio de Frazão P et al. (2010) muestra mayor riesgo de tenosinovitis en la raza blanca con una prevalencia del 60,8\% y una OR $=1,46$ (IC95\% 1,21-1,77) respecto a las personas de otra raza. ${ }^{19}$ 
Nivel socioeconómico: Frazão P et al. (2010) encontró mayor riesgo de padecer tenosinovitis en aquellos trabajadores que tenían mayor salario mensual (OR = 1,87(IC95\% 1,40-2,49), y también para los que tuvieron más de 11 años de formación $\left(\mathrm{OR}=1,54\right.$ (IC95\% 1,10-2,16), ${ }^{19}$ con resultados similares a los encontrados por D'Almeida. $(2008) .{ }^{21}$

Otras patologías de base: De la Parra et al (2008) encontraron una asociación entre la presencia de determinados factores de riesgo como la diabetes y la obesidad con la aparición de tenosivitis estenosante con una OR = 3,72 (IC95\% 2,43-5,70) y una $\mathrm{OR}=1,49$ (IC95\% 1,02-2,19) respectivamente, ${ }^{22}$ resultados similares a los obtenidos por Roquelaure et al. (2009) con $\mathrm{OR}=2,2 ; \mathrm{p}=0,014$ para la obesidad y $\mathrm{OR}=4,9 ; \mathrm{p}=0,001$ para la diabetes. ${ }^{13}$ En relación a la depresión Frazão P et al. (2010) obtiene una OR = 3,04 (IC95\% 2,43 - 3,81) ${ }^{19}$, la existencia de una lesión previa como factor de riesgo individual y los antecedentes de haber sido sometido a alguna intervención quirúrgica también se mostraron como factores riesgo para el desarrollo de tenosinovitis como se demuestra en los estudios de Waters et al. (2007) ${ }^{16}$ y los de Healy et al. (2011). ${ }^{17}$

\section{Trastornos musculoesqueléticos asociados a tenosinovitis}

Síndrome del manguito de los rotadores: $J u Y Y$ et al. (2011) ${ }^{18}$ en un estudio transversal con 146 entrenadores de atletismo de Taiwan observa que el 26\% de los entrenadores de atletismo en Taiwán presentaban patología del hombro mientras que Roquelaure $Y$. et al. (2009) encuentra que el 7,4\% de los trabajadores franceses tienen síndrome del manguito de los rotadores ${ }^{13}$.

Otros autores como Rozenfeld $V$ et al. (2010) y Chung Y. et al. (2013) obtienen resultados estadísticamente significativos con unas OR mayores de 1 indicando que hay una asociación entre la enfermedad y la exposición ocupacional. ${ }^{15,20}$ Rozenfeld $V$ et al. (2010) observan una asociación entre la tenosinovitis con la el padecimiento de otros trastornos musculoesqueléticos en fisioterapeutas israelíes con una OR=1,04 ${ }^{15}$ y Chung $Y$. et al. (2013) con una OR = 4,33 (IC 95\% 2,51-7,47) entre las enfermeras taiwanesas. ${ }^{20}$

Enfermedad de De Quervain: Özdolap et al. (2013) concluye que la enfermedad de De Quervain es uno de los trastornos musculoesqueléticos más comunes entre los mineros del carbón, observando mayores tasas de prevalencia comparadas con oficinistas en una región del noroeste de Turquía $(\mathrm{p}=0,029) .{ }^{23}$ Laoopugsin et al. (2012) en su estudio entre los empleados de una fábrica en Tailandia observa que la tasa de prevalencia de la enfermedad de De Quervain es del 13,6\% ${ }^{10}$.

Autores como Petit Le Manac'b et al. (2011) en un estudio transversal con 3.710 trabajadores encontraron una prevalencia de $1,2 \%,{ }^{12}$ similar, aunque algo superior a la que describe Healy K.A et al. (2011) con una tasa de prevalencia de 0,8\%. ${ }^{17}$

Los resultados del estudio de Rozenfeld V. et al. (2010) también apoyan la enfermedad de De Quervain como una de las tenosinovitis más frecuentes, al realizar un estudio de trastornos musculoesqueléticos en fisioterapeutas presentando una OR = 1,11(IC95\% 1,5$1,17)^{15}$ al realizar el tratamiento manual.

Dedo en gatillo: De la Parra-Márquez et al. (2008) describen altas tasas de prevalencia por lo que la consideran una patología frecuente entre las enfermedades osteotendinosas y en un estudio de casos y controles con 500 pacientes observan que se presenta asociada a la diabetes con una OR = 3,72 (IC95\% 2,43-5,70), a la obesidad con una OR = 1,49 ( IC95\% 1,02-2,19) y a determinadas actividades laborales como el trabajo de secretaria con una OR $=2,74$ (IC95\% 1,38-5,52), al de costurera con una OR $=4,8$ (IC95\% 1,3-21,6) y a la de ama de casa con una OR = 2,44 (IC95\% 1,62-3,69). ${ }^{22}$ Otros estudios también coinciden que se trata de una patología de las más frecuentes, describiendo tasas elevadas de prevalencia entre diferentes trabajadores, como es el caso de los autores Chung Y.-C. et al. (2013) ${ }^{20}$ y Laoopugsin et al. (2012) ${ }^{10}$. 
Epicondilitis lateral y medial: Chung Y.-C et al. (2013) en un estudio de casos y controles con 3.914 enfermeras taiwanesas encontró que la epicondilitis medial era la segunda patología tendinosa más frecuente después del síndrome del manguito de los rotadores. La epicondilitis medial presentaba una OR = 3,37 (IC95\% 1,49-7,63) y la epicondilitis lateral una OR $=2,16(\text { IC95\% 1,23-3,79 })^{20}$ Özdolap $S$ et al. (2013) en un estudio de caso y controles con 120 trabajadores de una región noroeste de Turquía entre ellos 80 mineros del carbón, observó que es una patología prevalente $(\mathrm{p}=0,024)^{23}$. Roquelaure $Y$ et al (2009) en un estudio transversal con 3.710 trabajadores observa que es la tercera patología más frecuente con una prevalencia de $2,6 \%$ detrás del síndrome del manguito de los rotadores ${ }^{13}$.

\section{DISCUSIÓN}

La literatura científica revisada parece indicar que existe una importante asociación entre determinados factores de riesgo laboral y la tenosinovitis de miembro superior.

Los factores de riesgos laborales asociados a diferentes trastornos osteotendinosos, identificados por los autores de los artículos incluidos en el estudio, concuerdan con los descritos en los estudios de campo desarrollados por la Occupational Safety and Health Administration (OSHA) en los que se definen los riesgos que se asocian estrechamente con el desarrollo de lesiones músculo-tendinosas ${ }^{1}$

En cuanto al riesgo psicosocial, dos estudios analizan la relación entre estos y la presencia de tenosinovitis, uno de ellos en cuanto a la depresión ${ }^{19}$ y otro en cuanto a la combinación de movimientos repetitivos con el estrés, ${ }^{16}$ encontrando una asociación estadísticamente significativa en relación a la depresión y comportándose el estrés como un factor de sinergia multiplicativa. Otros dos estudios no encuentran asociación entre los factores psicosociales con los trastornos musculoesqueléticos. ${ }^{12,14}$

Estudios previos a nuestro periodo de estudio, como los de Armstrong que en 1987, que estudiaba la asociación entre la tenosinovitis con la repetición de tareas ocupacionales que implican a la mano y el grado de fuerza de agarre, concluye que la combinación de la repetición, fuerza y postura presentan una fuerte asociación con la tenosinovitis. ${ }^{24}$

En un metaanálisis realizado por Stéphane Stabl et al. (2013) en el que evaluaron los riesgos laborales relacionados con la aparición de enfermedad De Quervain, se concluye que no es posible demostrar relación causa-efecto entre factores de riesgo laborales y enfermedad de De Quervain. ${ }^{26}$

En resumen, podíamos destacar que entre los principales factores de riesgo externos relacionados con las tenosinovitis, uno de los más importantes serían los movimientos repetitivos, seguidos de las posturas forzadas y el uso intensivo de herramientas manuales vibratorias. Entre los factores de riesgo individuales, el aumento de la edad supone un incremento del riesgo de desarrollar este tipo de patología, así como el ser mujer y de raza blanca también constituyen factores de riesgo importantes para el aparición de tenosinovitis y tendinopatías. Otros factores de riesgo como diabetes mellitus, obesidad y el haber tenido una lesión osteomuscular previa, también se encuentran relacionados con la enfermedad.

En cuanto a factores psicológicos la asociación entre trastornos musculoesqueléticos, la depresión parecen ser uno de los que pueden jugar un papel importante y el estrés podría actuar como factor potenciados a través de un efecto multiplicativo.

Entre los trastornos musculoesqueléticos asociados a tenosinovitis más prevalentes, podríamos señalar el síndrome del manguito de los rotadores, la enfermedad de De Quervain, el dedo en gatillo y las epicondilitis lateral y medial, aunque debido a los frecuentes factores multicausales que suelen acompañar la aparición de este tipo de afecciones, en muchas ocasiones se hace difícil lograr establecer una relación exclusiva de causalidad entre las tenosinovitis y las actividades laborales. 
Sólo se han encontrado tres estudios orientado a investigar la prevalencia el riesgo de padecer tenosinovitis en colonoscopistas, pese a que estos profesionales se encuentran entre la población de mayor riesgo de padecer enfermedad de De Quervain, ${ }^{27-30}$ por lo que se ha encontrado literatura reivindicando una mayor atención hacia este tipo de estudios con vistas a mejorar los diseños ergonómicos de los endoscopios, en los que para su manejo es frecuente tener que realizar maniobras repetitivas en posiciones forzadas, como se demuestra en uno de esos trabajos que realizó un estudio sobre la fuerza de presión y la carga de los músculos del antebrazo durante la colonoscopia y cuyos resultados ayudaron a plantear una segunda fase de investigación con una cohorte más amplia de gastroenterólogos para evaluar también las medidas subjetivas de tensión y fatiga musculoesquelética. Las mejoras ergonómicas en los endoscopios, podía suponer una de las mejores estrategias a largo plazo para reducir lesiones tendinosas en el personal sanitario que los maneja, asociado a otras medidas paralelas como adoptar pautas específicas para optimizar los sistemas de horarios de trabajo rotativos y el realizar ejercicios específicos para mejorar el trauma relacionado con la tarea.

Para finalizar, y como posibles factores de riesgo emergentes dentro de nuestra sociedad actual, algunas cuestiones relacionadas con los aspectos ergonómicos, los factores de riesgo psicosociales y el posible papel que puedan estar ejerciendo el uso de nuevas tecnologías como texting, whatsapping, videoconsolas, etc. se plantean como nuevos retos en el estudio de determinadas patologías osteotendinosas.

\section{REFERENCIAS BIBLIOGRÁFICAS}

1. European Union, European Agency for Safety and Health at Work. OSH in figures: work-related musculoskeletal disorders in the EU: facts and figures. Luxembourg: EUR-OP; 2010

2. International Labour Office. List of occupational diseases identification and recognition of occupational diseases: criteria for incorporating diseases in the ILO list of occupational diseases. [Internet]. Geneva: International Labour Office; 2010 [citado 26 de enero de 2015]. Recuperado a partir de: http://site.ebrary. com/id/10512127.

3. Houtman IL, Kompier MA. Trabajo y salud mental. Encicl Salud Segur En El Trab Madr Organ Int Trab. 2001;5-2.

4. Bevan S, Quadrello T, McGee R, Mahdon M, Vavrovsky A, Barham L. Fit for work? Musculoskeletal disorders in the European workforce. London: The Work Foundation, 2009. 2009.

5. Ley 31/1995, de 8 de noviembre, de Prevención de Riesgos Laborales. - leydeprevencionderiesgoslaborales. pdf [Internet]. [citado 25 de enero de 2015]. Recuperado a partir de: http://www.insht.es/InshtWeb/ Contenidos/Normativa/TextosLegales/LeyPrevencion/PDFs/leydeprevencionderiesgoslaborales.pdf.

6. BOE 302 de 19/12/2006 Sec 1 Pag 44487 a 44546 - A44487-44546.pdf [Internet]. [citado 25 de enero de 2015]. Recuperado a partir de: https://www.boe.es/boe/dias/2006/12/19/pdfs/A44487-44546.pdf.

7. Lista de enfermedades profesionales (revisada en 2010) identificación y reconocimiento de las enfermedades profesionales: criterios para incluir enfermedades en la lista de enfermedades profesionales de la OIT. [Internet]. Ginebra [Geneva]: Oficina International del Trabajo; 2010 [citado 29 de enero de 2015]. Recuperado a partir de: http://site.ebrary.com/id/10512084.

8. Zimmermann-Verdejo M. INFORME_EEPP2007-2012_JUNIO2013.pdf [Internet]. Departamento de Investigación e información Instituto Nacional de Seguridad e Higiene en el Trabajo; 2014 [citado 21 de enero de 2015]. Recuperado a partir de: http://www.oect.es/Observatorio/5\%20Estudios\%20tecnicos/ Otros\%20estudios\%20tecnicos/Publicado/Ficheros/INFORME_EEPP2007-2012_JUNIO2013.pdf.

9. Seguridad Social:Estadísticas, Presupuestos y Estudios [Internet]. [citado 21 de enero de 2015]. Recuperado a partir de: http://www.seg-social.es/Internet_1/Estadistica/Est/Observatorio_de_las_Enfermedades_ Profesionales/cepross2k11/Partes_comunicados/Tablas/187281.

10. Laoopugsin $\mathrm{N}$, Laoopugsin $\mathrm{S}$. The study of work behaviours and risks for occupational overuse syndrome. Hand Surg Int J Devoted Hand Up Limb Surg Relat Res J Asia-Pac Fed Soc Surg Hand. 2012;17(2):205-12.

11. Roquelaure Y, LeManach AP, Ha C, Poisnel C, Bodin J, Descatha A, et al. Working in temporary employment and exposure to musculoskeletal constraints. Occup Med. 2012;kqs004.

12. Petit Le Manac'h A, Roquelaure Y, Ha C, Bodin J, Meyer G, Bigot F, et al. Risk factors for de Quervain's disease in a French working population. Scand J Work Environ Health. Septiembre de 2011;37(5):394401 
13. Roquelaure Y, Ha C, Rouillon C, Fouquet N, Leclerc A, Descatha A, et al. Risk factors for upper-extremity musculoskeletal disorders in the working population. Arthritis Rheum. 15 de octubre de 2009;61(10):1425-34

14. Bodin J, Ha C, Petit Le Manac'h A, Sérazin C, Descatha A, Leclerc A, et al. Risk factors for incidence of rotator cuff syndrome in a large working population. Scand J Work Environ Health. Septiembre de 2012;38(5):436-46.

15. Rozenfeld V, Ribak J, Danziger J, Tsamir J, Carmeli E. Prevalence, risk factors and preventive strategies in work-related musculoskeletal disorders among Israeli physical therapists. Physiother Res Int. 2010;15(3):176-84

16. Waters TR, Dick RB, Davis-Barkley J, Krieg EF. A cross-sectional study of risk factors for musculoskeletal symptoms in the workplace using data from the General Social Survey (GSS). J Occup Environ Med. 2007;49(2):172-84

17. Healy KA, Pak RW, Cleary RC, Colon-Herdman A, Bagley DH. Hand problems among endourologists. J Endourol Endourol Soc. diciembre de 2011;25(12):1915-20.

18. Ju Y-Y, Cheng H-YK, Hsieh Y-J, Fu L-L. Work-related musculoskeletal disorders in athletic trainer. J Occup Rehabil. junio de 2011;21(2):190-8.

19. Frazão P, Costa CM, de Almeida MF. Risks associated with tendinitis: effects from demographic, socioeconomic, and psychological status among Brazilian workers. Am J Ind Med. enero de 2010;53(1):72-9.

20. Chung Y-C, Hung C-T, Li S-F, Lee H-M, Wang S-G, Chang S-C, et al. Risk of musculoskeletal disorder among Taiwanese nurses cohort: a nationwide population-based study. BMC Musculoskelet Disord. 23 de abril de 2013;14(1):144

21. D' Almeida KW, Godard C, Leclerc A, Lahon G. Sickness absence for upper limb disorders in a French company. Occup Med. 2008;58(7):506-8.

22. De la Parra-Márquez ML, Tamez-Cavazos R, Zertuche-Cedillo L, Martínez-Pérez JJ, Velasco-Rodríguez V, Cisneros-Pérez V. [Risk factors associated with trigger finger. Case-control study]. Cir Cir. agosto de 2008;76(4):323-7.

23. Özdolap S, Emre U, Karamercan A, Sarikaya S, Köktürk F. Upper limb tendinitis and entrapment neuropathy in coal miners. Am J Ind Med. mayo de 2013;56(5):569-75.

24. Palmer KT, Harris EC, Coggon D. Compensating occupationally related tenosynovitis and epicondylitis: a literature review. Occup Med Oxf Engl. enero de 2007;57(1):67-74.

25. Putz-Anderson V, Bernard BP, Burt SE, Cole LL, Fairfield-Estill C, Fine LJ, et al. Musculoskeletal disorders and workplace factors. Natl Inst Occup Saf Health NIOSH [Internet]. 1997 [citado 29 de enero de 2015]; Recuperado a partir de: http://static-cdn.compliancetrainingonline.com/docs/97-141.pdf.

26. Stahl S, Vida D, Meisner C, Lotter O, Rothenberger J, Schaller H-E, et al. Systematic review and metaanalysis on the work-related cause of de Quervain tenosynovitis: a critical appraisal of its recognition as an occupational disease. Plast Reconstr Surg. diciembre de 2013;132(6):1479-91.

27. Hu C-T. The SET maneuvers for reducing left-hand strain while doing colonoscopy in a sitting position. Gastrointest Endosc. marzo de 2011;73(3):639-40.

28. Cappell MS. Extension of an excellent pilot study to a quantitative analysis of the pathophysiology of de Quervain disease associated with colonoscopy. Gastrointest Endosc. noviembre de 2009;70(5):1050; author reply 1050-1.

29. Guelrud M. Improving control of the colonoscope: the "pinkie maneuver». Gastrointest Endosc. febrero de 2008;67(2):388-9; author reply 389.

30. Rex DK. Maximizing control of tip deflection with sound ergonomics: the "left hand shaft grip". Gastrointest Endosc. mayo de 2007;65(6):950-1; author reply 951. 